\title{
Hall Effect on Bénard Convection of Compressible Viscoelastic Fluid through Porous Medium
}

\author{
Mahinder Singh ${ }^{1}$ and Chander Bhan Mehta ${ }^{2}$ \\ ${ }^{1}$ Department of Mathematics, Government Post Graduate College Seema (Rohru), Shimla District, Himachal Pradesh 171207, India \\ ${ }^{2}$ Department of Mathematics, Centre of Excellence, Government Degree College Sanjauli, Shimla District, \\ Himachal Pradesh 171006, India
}

Correspondence should be addressed to Mahinder Singh; mahinder_singh91@rediffmail.in

Received 17 April 2013; Revised 26 July 2013; Accepted 30 July 2013

Academic Editor: Amy Shen

Copyright (C) 2013 M. Singh and C. B. Mehta. This is an open access article distributed under the Creative Commons Attribution License, which permits unrestricted use, distribution, and reproduction in any medium, provided the original work is properly cited.

\begin{abstract}
An investigation made on the effect of Hall currents on thermal instability of a compressible Walter's $B^{\prime}$ elasticoviscous fluid through porous medium is considered. The analysis is carried out within the framework of linear stability theory and normal mode technique. For the case of stationary convection, Hall currents and compressibility have postponed the onset of convection through porous medium. Moreover, medium permeability hasten postpone the onset of convection, and magnetic field has duel character on the onset of convection. The critical Rayleigh numbers and the wave numbers of the associated disturbances for the onset of instability as stationary convection have been obtained and the behavior of various parameters on critical thermal Rayleigh numbers has been depicted graphically. The magnetic field, Hall currents found to introduce oscillatory modes, in the absence of these effects the principle of exchange of stabilities is valid.
\end{abstract}

\section{Introduction}

The theoretical and experimental results of the onset of thermal instability (Bénard convection) under varying assumptions of hydrodynamic and hydromantic stability have been discussed in a treatise by Chandrasekhar [1] in his celebrated monograph. If an electric field is applied at right angles to the magnetic field, the whole current will not flow along the electric field. This tendency of the electric current of flow across an electric field in the presence of a magnetic field is called Hall current effect. The Hall effect is likely to be important in many geophysical and astrophysical situations as well as in flows of laboratory plasma. The use of the Boussinesq approximation has been made throughout, which states that the variations of density in the equations of motion can safely be ignored everywhere except in its association with the external force. It has been shown by Sato [2] and Tani [3] that inclusion of Hall currents gave rise to a cross flow, that is, a flow at right angle to the primary flow through a channel in the presence of a transverse magnetic field. In particular, Tani [3] has found that Hall effect produces a cross-flow of double-swirl pattern in incompressible flow through a straight channel with arbitrary cross-section. This breakdown of the primary flow and formation of secondary flow may be presumably attributed to the inherent instability of the primary flow in the presence of Hall current. Sato [2] has pointed out that even if the distribution of the primary flow velocity is stable to external disturbances, the whole layer may become turbulent if the distribution of the cross flow is unstable. Sherman and Sutton [4] have considered the effect of Hall current on the efficiency of a magnetofluid generator. The effect of Hall current on the thermal instability of a horizontal layer of electrically conducting fluid has been studied by Gupta [5].

Hall currents are effects whereby a conductor carrying an electric current perpendicular to an applied magnetic field develops a voltage gradient which is transverse to both the current and the magnetic field. It was discovered by Hall in 1879, while he was working on his doctoral degree at Johns Hopkins University at Baltimore, Maryland. The Hall effect has again become an active area of research with the discovery of the quantized Hall effect by Klaus von Klitzing for which he 
was bestowed with Nobel prize of physics in 1985. In ionized gases (plasmas), where the magnetic field is very strong and effects the electrical conductivity, cannot be Hall currents.

In the aforementioned studies, the medium has been considered to be nonporous. The development of geothermal power resources has increased general interest in the properties of convection in porous media. The effect of a magnetic field on the stability of such a flow is of interest in geophysics particularly in the study of Earth's core where the Earth's mantle which consists of conducting fluid behaves like a porous medium which can become convectively unstable as a result of differential diffusion. The other application of the results of a magnetic field is in the study of the stability of a convective flow in the geothermal region.

When the fluids are compressible, the equations governing the system become quite complicated to simplify. Boussinesq tried to justify the approximation for compressible fluids when the density variations arise principally from thermal effects. Spiegel and Veronis [6] have simplified the set of equations governing the flow of compressible fluids under the following assumptions.

(a) The depth of the fluid layer is much less than the scale height, as defined by them.

(b) The fluctuations in temperature, density, and pressure, introduced due to motion, do not exceed their total static variations.

Under the previous approximations, the flow equations are the same as those for incompressible fluids, except that the static temperature gradient is replaced by its excess over the adiabatic one and $C_{v}$ is replaced by $C_{p}$.

Chandra [7] observed a contradiction between the theory and experiment for the onset of convection in fluids heated from below. He performed the experiment in an air layer and found that the instability depended on the depth of the layer. Scanlon and Segel [8] have considered the effects of suspended particles on the onset of Bénard convection and found that the critical Rayleigh number is reduced because of the heat capacity of the particles. The suspended particles were thus found to destabilize the layer. The fluids have been considered to be Newtonian, and the medium has been considered to be nonporous in all the previous studies.

One class of elastico-viscous fluids is Walters fluid (model $B^{\prime}$ ), which is not characterized by Maxwell's/Oldroyd's, constitutive relation. When the fluid permeates a porous material, the gross effect is represented by Darcy's law. As a result of this macroscopic law, the usual viscous and viscoelastic terms in the equation of Walters' fluid (model $B^{\prime}$ ) motion are replaced by the resistance terms $\left[-\left(1 / k_{1}\right)\left(\mu-\mu^{\prime}(\partial / \partial t)\right) \vec{q}\right]$, where $\mu$ and $\mu^{\prime}$ are the viscosity and viscoelasticity of Walters' fluid (model $B^{\prime}$ ), $k_{1}$ is the medium permeability and $\vec{q}$ is the Darcian filter velocity of the fluid.

The flow through porous media is of considerable interest for petroleum engineers and geophysical fluid dynamicists. A great number of applications in geophysics may be found in the books by Phillips [9], Ingham and Pop [10], and Nield and Bejan [11]. The scientific importance of the field has also increased because hydrothermal circulation is the dominant heat transfer mechanism in young oceanic crust (Lister [12]).
Generally it is accepted that comets consisting of a dusty "snowball" of a mixture of frozen gasses, which is in the process of their journey, change from solid to gas and vice versa. The physical properties of comets that meteoroids, and interplanetary dust strongly suggest the importance of porosity in astrophysical context have been studied by McDonnell [13].

The stability of two superposed conducting Walters' $B^{\prime}$ elastico-viscous fluids in hydromagnetics has been studied by Sharma and Kumar [14] and whereas the instability of streaming Walters' viscoelastic fluid $B^{\prime}$ in porous medium has been considered by Sharma [15], Sunil and Chand [16]. Sunil et al. $[17,18]$ studied the Hall effect on thermosolutal instability of Rivlin-Ericksen and Walters' (model $B^{\prime}$ ) fluid in porous medium. In one study of Singh [19], Hall current effect on thermosolutal instability in a viscoelastic fluid flowing through porous medium and magnetic field stable solute gradient are found to have stabilizing effects on the system, whereas Hall current and medium permeability have a destabilizing effect on the system. The sufficient conditions for the nonexistence of overstability have also obtained. In the one another study, Singh and Kumar [20], hydrodynamic and hydromagnetic stability of two stratified Walter's $B^{\prime}$ elasticoviscous superposed fluids, where system is stable for stable stratification and unstable for unstable stratification and in case of horizontal magnetic field system having stabilizing effect for unstable stratification, is in contrast to the stability of two superposed Newtonian fluids, where the system is stable for stable stratifications. Gupta et al. [21] have studied thermal convection of dusty compressible Rivlin-Ericksen viscoelastic fluid with Hall currents and found that compressibility and magnetic field postpone the onset of convection, whereas Hall current and suspended particles hasten the onset of convection.

During the survey it has been noticed that Hall effects are completely neglected from the studies of compressible elastico-viscous fluid through porous medium. Keeping in mind the importance of Hall currents, porous medium and compressibility, in elastico-viscous fluid, motivated us to go on detailed study of Walter's $B^{\prime}$ fluid heated from below through porous medium. We have already studied earlier some problems on Hall current effect with porous as well as nonporous medium and suspended particles found the useful and interesting results, so compressible thermal instability problem of Walter's $B^{\prime}$ fluid with Hall currents effects through porous medium studied by us here.

\section{Mathematical Formulation of the Problem}

We have considered an infinite, horizontal, and compressible electrically conducting Walter's $B^{\prime}$ fluid permeated with porous medium in Hall current effect, bounded by the planes $z=0$ and $z=d$, as shown in Figure 1. This layer is heated from below so that temperature at bottom (at $z=0$ ) and the upper layer (at $z=d$ ) is $T_{0}$ and $T_{d}$, respectively, and a uniform temperature gradient $\beta(=|d T / d z|)$ is maintained. A uniform vertical magnetic field intensity $\vec{H}(0,0, H)$ and gravity force $\vec{g}(0,0,-g)$ pervade the system. 


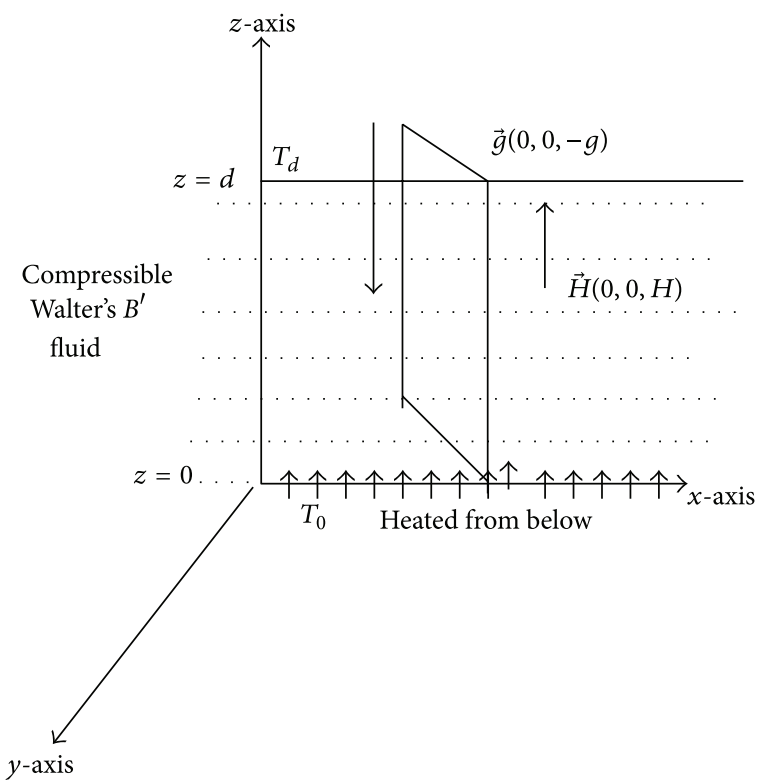

FIgURE 1: Geometrical configuration.

Let $p, \rho, T, \alpha, g, \eta, \mu_{e}$, and $\vec{q}(u, v, w)$ denote, respectively, the fluid pressure, density, temperature, thermal coefficient of expansion, gravitational acceleration, resistivity, magnetic permeability, and fluid velocity. Using Spiegel and Veronis' [6] assumptions, the flow equations for compressible fluids are found to be the same as those for incompressible fluids except that in the equation of heat conduction, the temperature gradient $\beta$ is replaced by its excess over the adiabatic; that is, $\left(\beta-g / c_{p}\right)$. The equations expressing the conservation of momentum, mass, temperature, and equation of state of Walters' (Model $B^{\prime}$ ) fluid are

$$
\begin{gathered}
\frac{1}{\epsilon}\left[\frac{\partial \vec{q}}{\partial t}+\frac{1}{\epsilon}(\vec{q} \cdot \nabla) \vec{q}\right] \\
=-\frac{1}{\rho_{m}} \nabla p+\vec{g}\left(1+\frac{\delta \rho}{\rho_{m}}\right)-\frac{1}{k_{1}}\left(\nu-\nu^{\prime} \frac{\partial}{\partial t}\right) \vec{q} \\
+\frac{\mu_{e}}{4 \pi \rho_{m}}(\nabla \times \vec{H}) \times \vec{H}, \\
\nabla \cdot \vec{q}=0, \\
E \frac{\partial T}{\partial t}+(\vec{q} \cdot \nabla) T=\left(\beta-\frac{g}{c_{p}}\right) w+\kappa \nabla^{2} T, \\
\rho=\rho_{m}\left[1-\alpha\left(T-T_{0}\right)\right] .
\end{gathered}
$$

The magnetic permeability $\mu_{e}$, the kinematic viscosity $v$, the kinematic viscoelasticity $v^{\prime}$, and the thermal diffusivity $\kappa$ are all assumed to be constants. Maxwell's equations relevant to the problems are

$$
\begin{gathered}
\epsilon \frac{d \vec{H}}{d t}=(\vec{H} \cdot \nabla) \vec{q}+\epsilon \eta \nabla^{2} \vec{H}-\frac{c \epsilon}{4 \pi N e} \nabla \times[(\nabla \times \vec{H} \times \vec{H})], \\
\nabla \cdot \vec{H}=0,
\end{gathered}
$$

where $d / d t=\partial / \partial t+\epsilon^{-1} \vec{q} \cdot \nabla$ stands for convective derivative.
Here $E=\epsilon+(1-\epsilon)\left(\rho_{s} c_{s} / \rho_{m} c_{f}\right)$ is a constant, and $E^{\prime}$ is a constant analogous to $E$ but corresponding to solute rather than heat. $\rho_{s}, c_{s}$ and $\rho_{m}, c_{f}$ stand for density and heat capacity of solid (porous matrix) material and fluid, respectively. The steady state solution is

$$
\begin{gathered}
\vec{q}=(0,0,0), \quad T=-\beta z+T_{0}, \\
\rho=\rho_{m}\left(1+\alpha \beta z-\alpha^{\prime} \beta^{\prime} z\right) .
\end{gathered}
$$

Let $\delta \rho, \delta p, \theta, \vec{h}\left(h_{x}, h_{y}, h_{z}\right)$, and $\vec{q}(u, v, w)$ denote, respectively, the perturbations in density $\rho$, pressure $p$, temperature $T$, magnetic field $\vec{H}(0,0, H)$, and filter velocity (zero initially). Then the linearized hydromagnetic perturbation equations through porous medium (Joseph [22], Walter's [23], Sherman and Sutton [4], and Spiegel and Veronis [6]), relevant to the problem, are

$$
\begin{gathered}
\left(\frac{1}{\epsilon}\right) \frac{\partial \vec{q}}{\partial t}=-\nabla\left(\frac{\delta p}{\rho_{m}}\right)+\vec{g} \frac{\delta \rho}{\rho_{m}}-\frac{1}{k_{1}}\left(v-v^{\prime} \frac{\partial}{\partial t}\right) \vec{q} \\
+\frac{\mu_{e}}{4 \pi \rho_{m}}(\nabla \times \vec{h}) \times \vec{H}, \\
\nabla \cdot \vec{q}=0, \\
\epsilon \frac{\partial \vec{h}}{\partial t}=(\vec{H} \cdot \nabla) \vec{q}+\epsilon \eta \nabla^{2} \vec{h}-\frac{c \epsilon}{4 \pi N e \eta} \nabla \times[(\nabla \times \vec{h}) \times \vec{H}], \\
\nabla \cdot \vec{h}=0, \\
E \frac{\partial \theta}{\partial t}=\left(\beta-\frac{g}{c_{p}}\right) w+\kappa \nabla^{2} \theta .
\end{gathered}
$$

And change in density $\delta \rho$ caused by perturbation $\theta$ in temperature is given by

$$
\delta \rho=-\alpha \rho_{m} \theta,
$$

where $\alpha$ is the coefficient of thermal expansion.

Writing (4) in scalar form, using (5), and eliminating $u, v$, $h_{x}, h_{y}$, and $\delta p$ between them, we obtain

$$
\begin{gathered}
\frac{1}{\epsilon} \frac{\partial}{\partial t} \nabla^{2} w=g \alpha\left(\frac{\partial^{2}}{\partial x^{2}}+\frac{\partial^{2}}{\partial y^{2}}\right) \theta-\frac{1}{k_{1}}\left(v-v^{\prime} \frac{\partial}{\partial t}\right) \nabla^{2} w \\
+\frac{\mu_{e} H}{4 \pi \rho_{m}} \nabla^{2} \frac{\partial h_{z}}{\partial z}, \\
\epsilon \frac{\partial h_{z}}{\partial t}=H \frac{\partial w}{\partial z}+\epsilon \eta \nabla^{2} h_{z}-\frac{c H \epsilon}{4 \pi N e} \frac{\partial \xi}{\partial z}, \\
\epsilon \frac{\partial \xi}{\partial t}=H \frac{\partial \varsigma}{\partial z}+\epsilon \eta \nabla^{2} \xi+\frac{c H \epsilon}{4 \pi N e} \nabla^{2} \frac{\partial h_{z}}{\partial z}, \\
\frac{1}{\epsilon} \frac{\partial \varsigma}{\partial t}=-\frac{1}{k_{1}}\left(v-v^{\prime} \frac{\partial}{\partial t}\right) \varsigma+\frac{\mu_{e} H}{4 \pi \rho_{m}} \frac{\partial \xi}{\partial z}, \\
E \frac{\partial \theta}{\partial t}=\left(\beta-\frac{g}{c_{p}}\right) w+\kappa \nabla^{2} \theta,
\end{gathered}
$$


where $\nabla^{2}=\partial^{2} / \partial x^{2}+\partial^{2} / \partial y^{2}+\partial^{2} / \partial z^{2} \cdot \varsigma=\partial v / \partial x-\partial u / \partial y$ and $\xi=(\partial / \partial x) h_{y}-(\partial / \partial y) h_{x}$ denote the $z$-components of vorticity and current density, respectively.

Consider the case in which both the boundaries are free, the medium adjoining the fluid is perfectly conducting and the temperatures at the boundaries are kept fixed. The case of two free boundaries is a little artificial, except in stellar atmospheres (Spiegel [24]) and in certain geophysical situations where it is most appropriate, but it allows us to have an analytical solution. It has been shown by Spiegel that the assumption of free boundary conditions is not a serious one, so in free boundary conditions, the vertical velocity temperature fluctuation horizontal stress and all vanish on the boundaries. The boundary conditions, appropriate to the problem, are (Chandrasekhar [1])

$$
\begin{gathered}
w=0, \quad \frac{\partial^{2} w}{\partial z^{2}}=0, \quad \theta=0, \quad \frac{\partial \varsigma}{\partial z}=0, \\
h_{z}=0 \quad \text { at } z=0, z=d .
\end{gathered}
$$

\section{The Dispersion Relation}

Analyzing the disturbances into normal modes, we seek solutions whose dependence on $x, y$, and $t$ is given by

$$
\begin{aligned}
{\left[w, \theta, h_{z}, \zeta, \xi\right]=} & {[W(z), \Theta(z), K(z), Z(z), X(z)] } \\
& \times \exp \left(i k_{x} x+i k_{y} y+n t\right),
\end{aligned}
$$

where $k_{x}, k_{y}$ are horizontal wave numbers, $k\left(=\sqrt{k_{x}^{2}+k_{y}^{2}}\right)$ is the resultant wave number, and $n$ is a complex constant. Using the dimensionless variables $a=k d, \sigma=n d^{2} / \nu, p_{1}=v / \kappa$, $p_{2}=v / \eta, P_{l}=k_{1} / d^{2}, F=v^{\prime} / d^{2}, x^{*}=x / d, y^{*}=y / d, z^{*}=$ $z / d$, and $D=d / d z^{*}$ and removing the stars for convenience, (5)-(6) with the help of (8) become

$$
\begin{gathered}
{\left[\frac{\sigma}{\epsilon}+\frac{1-\sigma F}{P_{l}}\right]\left(D^{2}-a^{2}\right) W+\frac{g \alpha d^{2} a^{2}}{\nu} \Theta} \\
-\frac{\mu_{e} H d}{4 \pi \rho_{m} \nu}\left(D^{2}-a^{2}\right) D K=0, \\
\left(\frac{\sigma}{\epsilon}+\frac{1-\sigma F}{P_{l}}\right) Z=\frac{\mu_{e} H d}{4 \pi \rho_{m} \nu} D X, \\
\left(D^{2}-a^{2}-p_{2} \sigma\right) K=-\left(\frac{H d}{\epsilon \eta}\right) D W+\frac{c H d}{4 \pi N e \eta} D X, \\
\left(D^{2}-a^{2}-p_{2} \sigma\right) X \quad-\left(\frac{H d}{\epsilon \eta}\right) D Z-\frac{c H}{4 \pi N e \eta d}\left(D^{2}-a^{2}\right) D K, \\
\left(D^{2}-a^{2}-E p_{1} \sigma\right) \Theta=-\left(\frac{G-1}{G}\right) \frac{\beta d^{2}}{\kappa} W .
\end{gathered}
$$

From boundary conditions (7), using expression (8), we have

$$
\begin{array}{r}
W=D^{2} W=0, \quad \Theta=0, \quad D Z=0, \quad K=0 \\
\text { at } z=0, z=1 .
\end{array}
$$

Eliminating $\Theta, K, Z$, and $X$ from (9), we obtain

$$
\begin{aligned}
\left(\frac{\sigma}{\epsilon}+\right. & \left.\frac{1-\sigma F}{P_{l}}\right)\left(D^{2}-a^{2}\right)\left(D^{2}-a^{2}-E p_{1} \sigma\right) \\
\times & {\left[\left(\frac{\sigma}{\epsilon}+\frac{1-\sigma F}{P_{l}}\right)\left(D^{2}-a^{2}-p_{2} \sigma\right)^{2}\right.} \\
& +\frac{Q}{\epsilon}\left(D^{2}-a^{2}-p_{2} \sigma\right) D^{2} \\
& \left.-M\left(\frac{\sigma}{\epsilon}+\frac{1-\sigma F}{P_{l}}\right)\left(D^{2}-a^{2}\right) D^{2}\right] W \\
- & R a^{2}\left(\frac{G-1}{G}\right) \\
\times & {\left[\left(\frac{\sigma}{\epsilon}+\frac{1-\sigma F}{P_{l}}\right)\left(D^{2}-a^{2}-p_{2} \sigma\right)^{2}\right.} \\
& +\frac{Q}{\epsilon}\left(D^{2}-a^{2}-p_{2} \sigma\right) D^{2} \\
& \left.-M\left(\frac{\sigma}{\epsilon}+\frac{1-\sigma F}{P_{l}}\right)\left(D^{2}-a^{2}\right) D^{2}\right] W \\
+ & \frac{Q}{\epsilon}\left(D^{2}-a^{2}\right)\left(D^{2}-a^{2}-E p_{1} \sigma\right) \\
\times & {\left[\left(\frac{\sigma}{\epsilon}+\frac{1-\sigma F}{P_{l}}\right)\left(D^{2}-a^{2}-p_{2} \sigma\right)+\frac{Q}{\epsilon} D^{2}\right] D^{2} W=0 . }
\end{aligned}
$$

Here $R=g \alpha \beta d^{4} / v \kappa$ is thermal Rayleigh number, $Q=$ $\mu_{e} H^{2} d^{2} / 4 \pi \rho_{m} \nu \eta$ is Chandrasekhar number, and $M=(\mathrm{cH} /$ $4 \pi N e \eta)^{2}$ is nondimensional number according to Hall currents.

It can be shown with the help of (9) and boundary conditions (10) that all the even order derivatives of $W$ vanish at the boundaries and hence the proper solution of (10) characterizing the lowest mode is

$$
W=W_{o} \sin \pi z
$$

where $W_{o}$ is a constant. Substituting (12) in (11) and letting $x=$ $a^{2} / \pi^{2}, R_{1}=R / \pi^{4}, Q_{1}=Q / \pi^{2}, i \sigma_{1}=\sigma / \pi^{2}$, and $P=\pi^{2} P_{l}$, we obtain the dispersion relation 


$$
\begin{aligned}
& R_{1} x=\left(\frac{G}{G-1}\right) \\
& \left\{\frac{1}{P}+i \sigma_{1}\left(\frac{1}{\epsilon}-\frac{\pi^{2} F}{P}\right)\right\}(1+x)\left(1+x+E i p_{1} \sigma_{1}\right) \\
& \times\left[\left\{\frac{1}{P}+i \sigma_{1}\left(\frac{1}{\epsilon}-\frac{\pi^{2} F}{P}\right)\right\}\left(1+x+i p_{2} \sigma_{1}\right)^{2}+\frac{Q_{1}}{\epsilon}\left(1+x+i p_{2} \sigma_{1}\right)-M\left\{\frac{1}{P}+i \sigma_{1}\left(\frac{1}{\epsilon}-\frac{\pi^{2} F}{P}\right)\right\}(1+x)\right] \\
& \times \frac{+\frac{Q}{\epsilon}(1+x)\left(1+x+i E p_{1} \sigma_{1}\right)\left[\left(1+x+i p_{2} \sigma_{1}\right) \times\left\{\frac{1}{P}+i \sigma_{1}\left(\frac{1}{\epsilon}-\frac{\pi^{2} F}{P}\right)\right\}+\frac{Q_{1}}{\epsilon}\right]}{(1)} \\
& \left(1+x+i p_{2} \sigma_{1}\right)^{2}\left\{\frac{1}{P}+i \sigma_{1}\left(\frac{1}{\epsilon}-\frac{\pi^{2} F}{P}\right)\right\}+\frac{Q_{1}}{\epsilon}\left(1+x+i p_{2} \sigma_{1}\right)-M(1+x)\left\{\frac{1}{P}+i \sigma_{1}\left(\frac{1}{\epsilon}-\frac{\pi^{2} F}{P}\right)\right\} .
\end{aligned}
$$

\section{The Stationary Convection}

For the case of stationary convection $\sigma=0$, and (13) reduces to

$$
\begin{aligned}
R_{1}= & \left(\frac{G}{G-1}\right)\left(\frac{1+x}{x}\right) \\
& \times \frac{\left((1+x) / P+Q_{1} / \epsilon\right)^{2}-M(1+x) / P^{2}}{(1+x) / P+Q_{1} / \epsilon-M / P} .
\end{aligned}
$$

In order to investigate the effects of Hall current, medium permeability, and magnetic field, we examine the behaviour of $d R_{1} / d M, d R_{1} / d P$, and $d R_{1} / d Q_{1}$ analytically. Equation (14) yields

$$
\frac{d R_{1}}{d M}=\frac{G}{G-1} \frac{Q_{1}(1+x)}{\epsilon x P} \frac{\left((1+x) / P+Q_{1} / \epsilon\right)}{\left((1+x) / P+Q_{1} / \epsilon-M / P\right)^{2}},
$$

which is positive. The Hall current, therefore, had postpone the onset of thermal convection through porous medium for $G>1$. It is evident from (14) that

$$
\begin{gathered}
\frac{d R_{1}}{d P}=-\frac{G}{G-1} \frac{(1+x)}{x P^{2}} \frac{\left((1+x) / P^{2}\right)(1+x-M)^{2}+2 Q_{1}(1+x)(1+x+M) / \epsilon P+\left(Q_{1} / \epsilon\right)^{2}(1+x+M)}{\left((1+x-M) / P+Q_{1} / \epsilon\right)^{2}}, \\
\frac{d R_{1}}{d Q_{1}}=\frac{G}{G-1} \frac{(1+x)}{\epsilon x} \frac{\left((1+x) / P+Q_{1} / \epsilon\right)\left((1+x) / P+Q_{1} / \epsilon-2 M / P\right)+\left(M / P^{2}\right)(1+x)}{\left((1+x-M) / P+Q_{1} / \epsilon\right)^{2}},
\end{gathered}
$$

which imply that for $G>1$, medium permeability hasten postpone the onset of convection, where as magnetic field has postponed the onset of convection in Walters' $B^{\prime}$ elasticoviscous fluid through porous medium for $Q_{1}>(\epsilon / P)[2 M-$ $(1+x)]$ and hasten postpone the onset of convection, if $Q_{1}<(\epsilon / P)[2 M-(1+x)]$. Therefore, magnetic field has duel character in presence of Hall currents through porous medium. For fixed $P, Q_{1}$, and $M$, let $G$ (accounting for the compressibility effects) also, be kept fixed in (14). Then we find that

$$
\bar{R}_{c}=\left(\frac{G}{G-1}\right) R_{c},
$$

where $\bar{R}_{c}$ and $R_{c}$ denote, respectively, the critical Rayleigh numbers in the presence and absence of compressibility. Thus, the effect of compressibility is to postpone the onset of thermal instability. The cases $G<1$ and $G=1$ correspond to negative and infinite values of Rayleigh number which are not relevant in the present study. $G>1$ is relevant here.
The compressibility, therefore, has postponed the onset of convection.

\section{Graphical Results and Discussion}

The dispersion relation (14), in case of stationary convection, has been computed by concerning mathematical software. The results have been displayed graphically for various parameters of interest. The effects of these parameters especially Hall parameter, medium permeability, magnetic field, Rayleigh number with wave number have been studied. In Figure 2, Rayleigh number $R_{1}$ is plotted against wave number $x(=10-80)$, for different values of Hall parameter $M(=$ $10-40)$ and fixed values of medium permeability parameter $P=3, G=10$, magnetic field parameter $Q_{1}=100$, and $\epsilon=$ 0.5 . Here, we find that with the increase in the value of Hall current parameter, value of Rayleigh number is increased, showing that the Hall currents parameter has stabilizing effect on the system. 


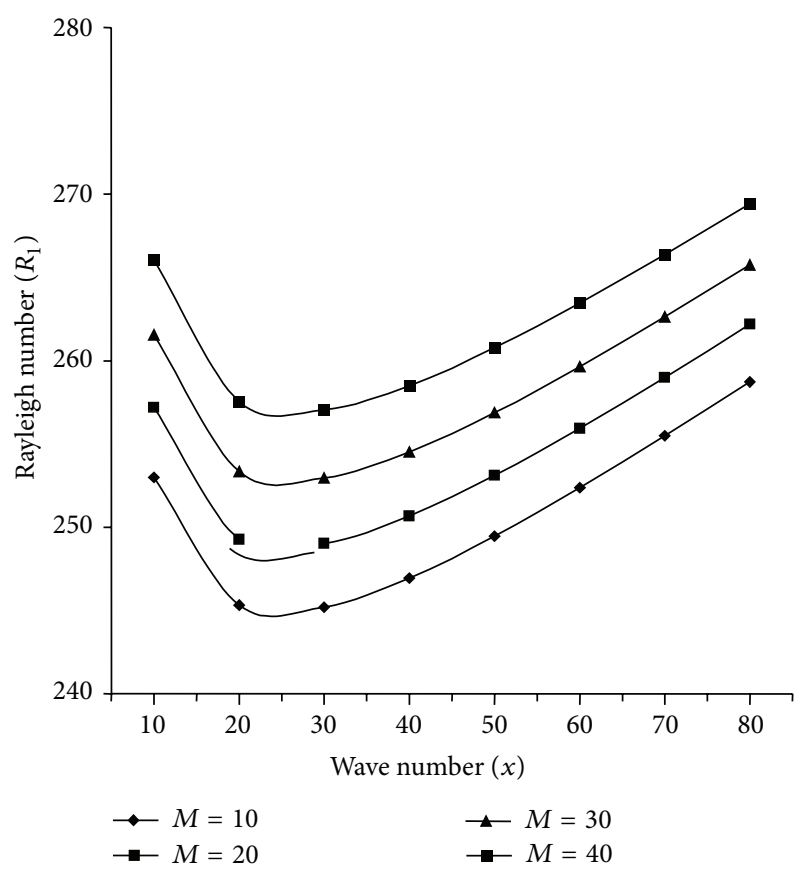

FIGURE 2: Variation of Rayleigh number $R_{1}$ against wave number $x$ for $P=3, G=10, Q_{1}=100$, and $\epsilon=0.5$.

In Figure 3, Rayleigh number $R_{1}$ is plotted against wave number $x(=1-5)$, and for different medium permeability parameter $P(=1,2,3,7)$, for fixed magnetic field parameter $Q_{1}=100$, Hall current parameter $M=10, G=$ 10 , and $\epsilon=0.5$ are considered. We find that as medium permeability $P$ increases, value of Rayleigh number $R_{1}$ decreases, which indicates the destabilizing effect of medium permeability.

In Figure 4, Rayleigh number $R_{1}$ is plotted against wave number $x(=10-80)$, and for different values of magnetic field parameter $Q_{1}(=10-40)$, for fixed values of medium permeability $P=3$, Hall current parameter $M=10, G=10$ and $\epsilon=0.5$ are considered. It is clear from the graph that with the increase in the value of magnetic field parameter, there is decrease as well as increase in the Rayleigh number $R_{1}$, implying the destabilizing as well as stabilizing effect on the system.

\section{The Case of Overstability}

In the present section, we discuss the possibility as to whether instability may occur as overstability. Since for overstability we wish to determine the critical Rayleigh number for the onset of instability via a state of pure oscillations, it will suffice to find conditions for which (13) will admit of solutions with $\sigma_{1}$ real. Equating real and imaginary parts of (13) and eliminating $R_{1}$ between them, we obtain

$$
A_{3} c_{1}^{3}+A_{2} c_{1}^{2}+A_{1} c_{1}+A_{o}=0
$$

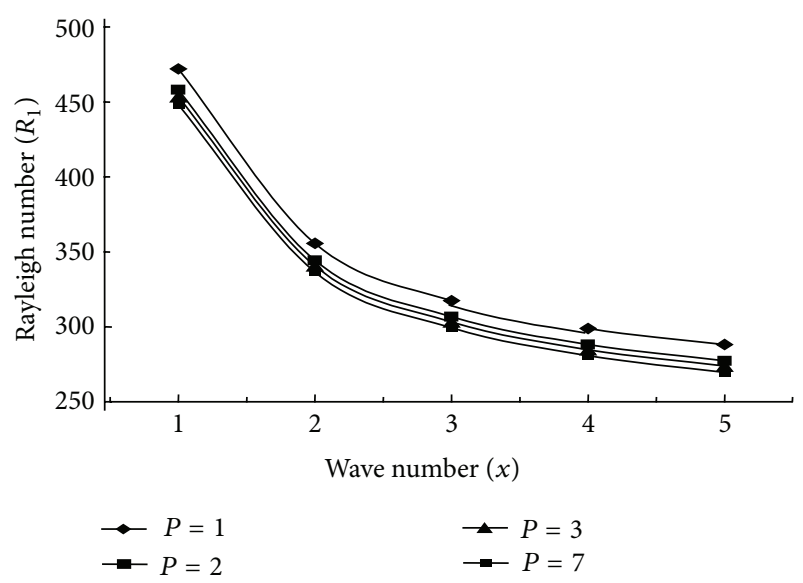

FIGURE 3: Variation of Rayleigh number $R_{1}$ against wave number $x$ for $Q_{1}=100, M=10, G=10$, and $\epsilon=0.5$.

where

$$
\begin{aligned}
& c_{1}=\sigma_{1}^{2}, \quad b=1+x \\
& A_{3}=p_{2}^{4}\left(\frac{1}{\epsilon}-\frac{\pi^{2} F}{P}\right)^{2}\left[\frac{E p_{1}}{P}+b\left(\frac{1}{\epsilon}-\frac{\pi^{2} F}{P}\right)\right], \\
& A_{o}=\frac{1}{P}\left(\frac{1}{\epsilon}-\frac{\pi^{2} F}{P}\right) b^{5} \\
&+\left[\frac{E p_{1}}{P}+\frac{2}{P}\left(\frac{Q_{1}}{\epsilon}-\frac{M}{P}\right)\left(\frac{1}{\epsilon}-\frac{\pi^{2} F}{P}\right)\right] b^{4} \\
&+ {\left[\left(\frac{Q_{1}}{\epsilon}-\frac{M}{P}\right)^{2}\left(\frac{1}{\epsilon}-\frac{\pi^{2} F}{P}\right)+\frac{2 E p_{1}}{P^{2}}\left(\frac{Q_{1}}{\epsilon}-\frac{M}{P}\right)\right.} \\
&\left.+\frac{Q_{1}}{\epsilon P^{2}}\left(E p_{1}-p_{2}\right)\right] b^{3} \\
&+ {\left[\frac{M Q_{1}}{\epsilon P^{2}}\left(3 E p_{1}+p_{2}\right)+\left(\frac{Q_{1}}{\epsilon}\right)^{2}\right.} \\
& \times\left\{\frac{2}{P}\left(E p_{1}-p_{2}\right)+E p_{1}-M\left(\frac{1}{\epsilon}-\frac{\pi^{2} F}{P}\right)\right\} \\
&+\left.+\frac{Q_{1}}{\epsilon}\right)^{2}\left[\frac{E p_{1} M}{P}+\frac{Q_{1}}{\epsilon}\left(E p_{1}-p_{2}\right)\right] b \\
&\left.+\frac{M^{2}}{P^{3}}\right] b^{2}
\end{aligned}
$$

The three values of $c_{1}, \sigma_{1}$ being real, are positive. The product of the roots of (18) is $-A_{0} / A_{3}$, and if this is to be positive then 


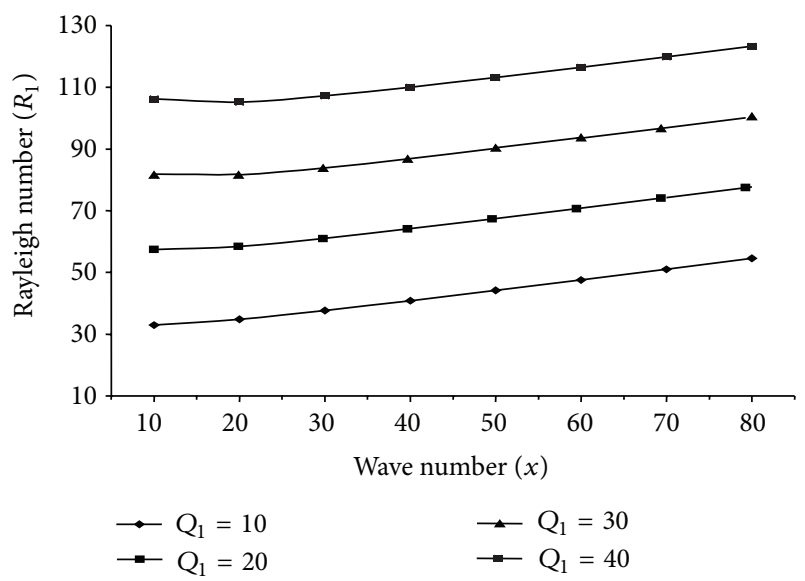

FIGURE 4: Variation of Rayleigh number $R_{1}$ against wave number $x$ for $P=3, M=10, G=100$, and $\epsilon=0.5$.

$A_{0}<0$, since from (20), $A_{3}>0$ if $1 / \epsilon>\pi^{2} F / P$. Equation

(17) shows that this is clearly impossible if

$$
\frac{1}{\epsilon}>\frac{\pi^{2} F}{P}, \quad E p_{1}>p_{2}, \quad E p_{1}>M\left(\frac{1}{\epsilon}-\frac{\pi^{2} F}{P}\right)
$$

which imply that

$$
v^{\prime}<\frac{k_{1}}{\epsilon}, \quad E_{\kappa}^{v}>\max \left[\frac{v}{\eta},\left(\frac{c H}{4 \pi N e \eta}\right)^{2} \frac{k_{1}-v^{\prime} \epsilon}{k_{1} \epsilon}\right] .
$$

Thus, $v^{\prime}<k_{1} / \epsilon$ and $E(v / \kappa)>\max \left[v / \eta,(c H / 4 \pi N e \eta)^{2}\left(\left(k_{1}-\right.\right.\right.$ $\left.\left.\left.v^{\prime} \epsilon\right) / k_{1} \epsilon\right)\right]$ are sufficient conditions for the nonexistence of overstability, the violation of which does not necessarily imply the occurrence of overstability.

\section{Concluding Remarks}

Combined effect of various parameters; that is, magnetic field, compressibility, medium permeability, and hall currents effect has been investigated on thermal instability of a Walter's $B^{\prime}$ fluid. The principle concluding remarks are as the following.

(i) For the stationary convection. Walter's $B^{\prime}$ fluid behaves like an ordinary Newtonian fluid due to the vanishing of the viscoelastic parameter.

(ii) The presence of magnetic field (and therefore Hall currents) and medium permeability effects introduce oscillatory modes in the system; in the absence of these effects, the principle of exchange of stabilities is valid.

(iii) The sufficient conditions for the occurrence of overstability are $v^{\prime}<k_{1} / \epsilon$ and $E(v / \kappa)>\max [v / \eta,(c H /$ $\left.4 \pi N e \eta)^{2}\left(\left(k_{1}-v^{\prime} \epsilon\right) / k_{1} \epsilon\right)\right]$, violation of which does not necessarily imply the occurrence of overstability.

(iv) From (17), it is clear that effect of compressibility has postponed the onset of convection. (v) To investigate the effects of medium permeability, magnetic permeability, and Hall currents in compressible Walter's $B^{\prime}$ viscoelastic fluid, we examined the expressions $d R_{1} / d M, d R_{1} / d P$, and $d R_{1} / d Q_{1}$ analytically. Hall current effect has postponed the onset of convection and medium permeability hastened the onset of convection, where magnetic field has postponed the onset of convection as well as hastened the onset of convection.

\section{Nomenclature}

$g:$

$K:$

$k$ :

$k_{x}, k_{y}:$

$k_{1}$ :

$m:$

$N$ :

$n$ :

$p:$

$t:$

$\vec{u}$ :

$\vec{v}:$

$\vec{H}$ :

$\beta(=|d T / d z|):$

$N_{p_{1}}:$

$\mathrm{N}_{p_{2}}:$

$R=g \alpha \beta d^{4} / v \kappa:$

$Q=\mu_{e} H^{2} d^{2} / 4 \pi \rho_{m} \nu \eta:$

$M=(c H / 4 \pi N e \eta)^{2}$ :

$f:$

$\zeta:$

$\xi$

$N_{R}^{C}$ and $\overline{N_{R}^{C}}$ :
Acceleration due to gravity $\left(\mathrm{ms}^{-2}\right)$ Stoke's drag coefficient $\left(\mathrm{kg} \mathrm{s}^{-1}\right)$

Wave number $\left(\mathrm{m}^{-1}\right)$

Horizontal wave-numbers $\left(\mathrm{m}^{-1}\right)$

Medium permeability $\left(\mathrm{m}^{2}\right)$

Mass of single particle ( $\mathrm{g}$ )

Suspended particle number density $\left(\mathrm{m}^{-3}\right)$

Growth rate $\left(\mathrm{s}^{-1}\right)$

Fluid pressure $(\mathrm{Pa})$

Time (s)

Fluid velocity $\left(\mathrm{ms}^{-1}\right)$

Suspended particle velocity $\left(\mathrm{ms}^{-1}\right)$

Magnetic field intensity vector

having component $(0,0, H)(\mathrm{G})$

Steady adverse temperature gradient $\left(\mathrm{Km}^{-1}\right)$

Thermal Prandtl number (-) Magnetic Prandtl number (-) thermal Rayleigh number Chandrasekhar number

Nondimensional number according to Hall currents The mass fraction $Z$ Component of vorticity $Z$ Component of current density Critical Rayleigh numbers in the absence and presence of compressibility.

\section{Greek Letters}

$\epsilon:$ Medium porosity $\left(\mathrm{m}^{0} \mathrm{~s}^{0} \mathrm{k}^{0}\right)$

$\mu$ : Dynamic viscosity $\left(\mathrm{km}^{-1} \mathrm{~s}^{-1}\right)$

$\mu^{\prime}$ : Fluid viscoelasticity $\left(\mathrm{km}^{-1} \mathrm{~s}^{-1}\right)$

$\nu$ : Kinematic viscosity $\left(\mathrm{m}^{2} \mathrm{~s}^{-1}\right)$

$\nu^{\prime}$ : Kinematic viscoelasticity $\left(\mathrm{m}^{2} \mathrm{~s}^{-1}\right)$

$\rho$ : Density $\left(\mathrm{kg} \mathrm{m}^{-3}\right)$.

\section{Acknowledgment}

The authors are grateful to the referees for their technical comments and valuable suggestions, resulting in a significant improvement of the paper. 


\section{References}

[1] S. Chandrasekhar, Hydrodynamic and Hydromagnetic Stability, Dover Publications, New York, NY, USA, 1981.

[2] H. Sato, "The Hall effect in the viscous flow of ionized gas between parallel plates under transverse magnetic field," Journal of the Physical Society of Japan, vol. 16, no. 7, pp. 1427-1433, 1961.

[3] I. Tani, "Steady flow of conducting fluid in channels under transverse magnetic field with consideration of Hall Effect," Journal of Aerospace Science, vol. 29, pp. 297-305, 1962.

[4] A. Sherman and G. W. Sutton, Magnetohydrodynamics, Northwestern University Press, Evanston, Ill, USA, 1962.

[5] A. S. Gupta, "Hall effects on thermal instability", Revue Roumaine de Mathématique Pures et Appliquées, pp. 665-677, 1967.

[6] E. A. Spiegel and G. Veronis, "On the Boussinesq approximation for a compressible fluid," The Astrophysical Journal, vol. 131, pp. 442-447, 1960.

[7] K. Chandra, "Instability of fluids heated from below," Proceedings of the Royal Society A, vol. 164, pp. 231-242, 1938.

[8] J. W. Scanlon and L. A. Segel, "Some effects of suspended particles on the onset of Bénard convection," Physics of Fluids, vol. 16, no. 10, pp. 1573-1578, 1973.

[9] O. M. Phillips, Flow and Reaction in Permeable Rocks, Cambridge University Press, Cambridge, UK, 1991.

[10] D. B. Ingham and I. Pop, Transport Phenomena in Porous Medium, Pergamon Press, Oxford, UK, 1998.

[11] D. A. Nield and A. Bejan, Convection in Porous Medium, Springer, New York, NY, USA, 2nd edition, 1999.

[12] C. R. B. Lister, "On the thermal balance of a mid-ocean ridge," Geophysics Journal of the Royal Astronomical Society Continues, vol. 26, pp. 515-535, 1972.

[13] J. A. M. McDonnell, Cosmic Dust, John Wiley \& Sons, Toronto, Canada, 1978.

[14] R. C. Sharma and P. Kumar, "Rayleigh-Taylor instability of two superposed conducting Walter's $B^{\prime}$ elastico-viscous fluids in hydromagnetics," Proceedings of the National Academy of Sciences A, vol. 68, no. 2, pp. 151-161, 1998.

[15] R. C. Sharma, "MHD instability of rotating superposed fluids through porous medium," Acta Physica Academiae Scientiarum Hungaricae, vol. 42, no. 1, pp. 21-28, 1977.

[16] S. Sunil and T. Chand, "Rayleigh-Taylor instability of plasma in presence of a variable magnetic field and suspended particles in porous medium," Indian Journal of Physics, vol. 71, no. 1, pp. 95-105, 1997.

[17] S. Sunil, R. C. Sharma, and V. Sharma, "Stability of stratified Walter's $B^{\prime}$ visco-elastic fluid in stratified porous medium," Studia Geotechnica et Mechenica, vol. 261, no. 2, pp. 35-52, 2004.

[18] S. Sunil, R. C. Sharma, and S. Chand, "Hall effect on thermal instability of Rivlin-Ericksen fluid," Indian Journal of Pure and Applied Mathematics, vol. 31, no. 1, pp. 49-59, 2000.

[19] M. Singh, "Hall Current effect on thermosolutal instability in a visco-elastic fluid flowing in a porous medium," International Journal of Applied Mechanics and Engineering, vol. 16, no. 1, pp. 69-82, 2011.

[20] M. Singh and P. Kumar, "Hydrodynamic and hydromagnetic stability of two stratified Walter's $B^{\prime}$ elastico-viscous superposed fluids," International Journal of Applied Mechanics and Engineering, vol. 16, no. 1, p. 233, 2011.

[21] U. Gupta, P. Aggarwal, and R. K. Wanchoo, "Thermal convection of dusty compressible Rivlin-Ericksen viscoelastic fluid with Hall currents," Thermal Science, vol. 16, no. 1, pp. 177-191, 2012.

[22] D. D. Joseph, Stability of Fluid Motion II, Springer, New York, NY, USA, 1976.

[23] K. Walter's, “The solution of flow problems in case of materials with memory," Journal of Mecanique, vol. 1, pp. 469-479, 1962.

[24] E. A. Spiegel, "Conveive instability in a compressible atmosphere," Journal of Astrophysics, vol. 141, pp. 1068-1090, 1965. 

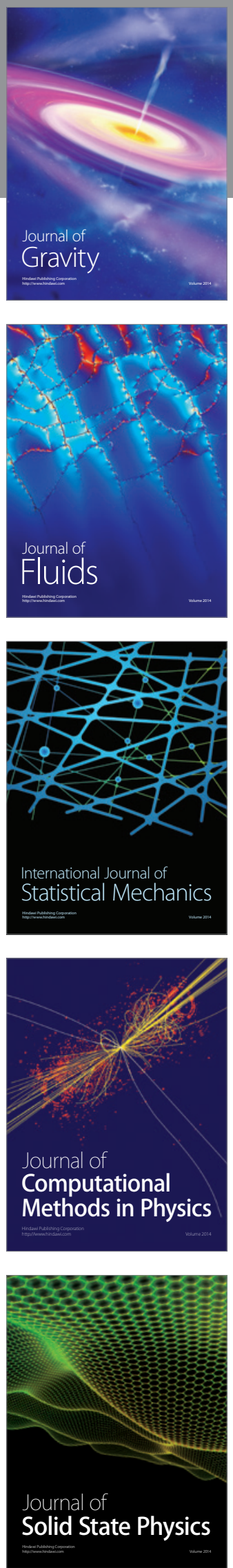

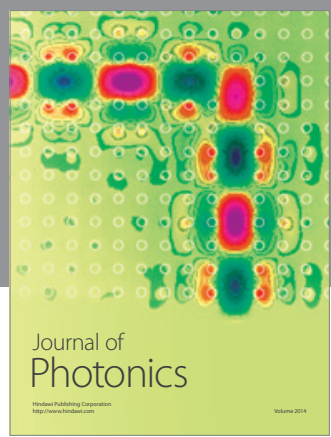

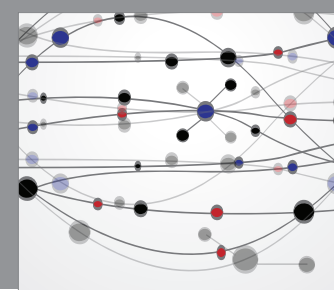

The Scientific World Journal

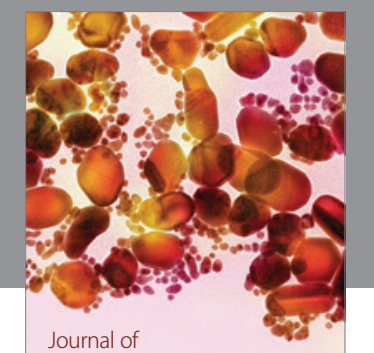

Soft Matter
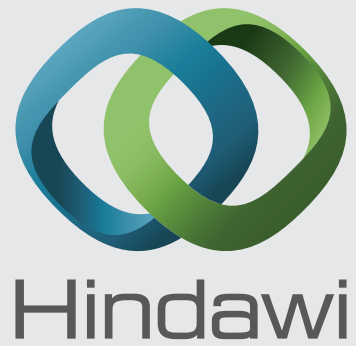

Submit your manuscripts at

http://www.hindawi.com
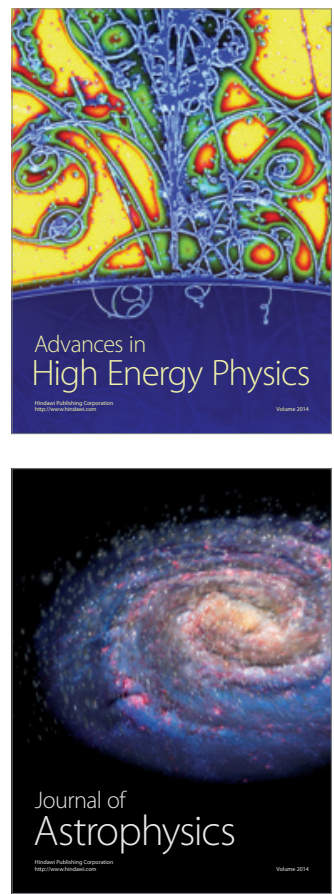
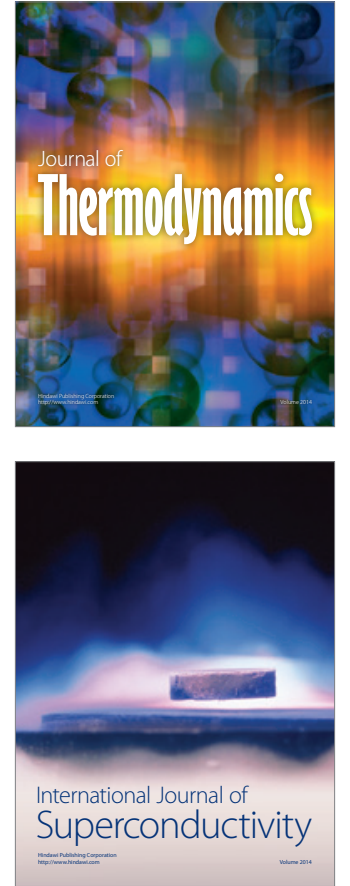
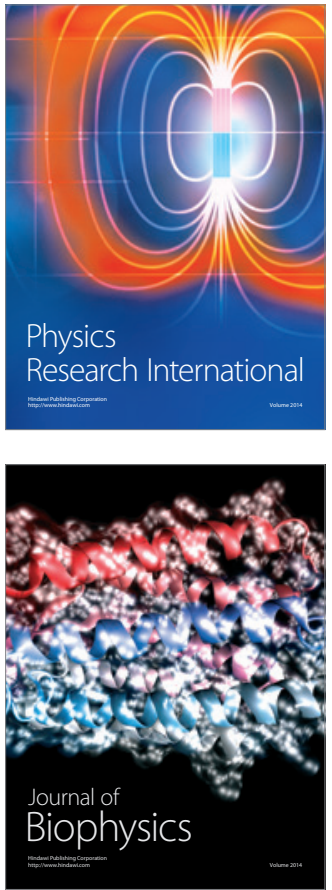
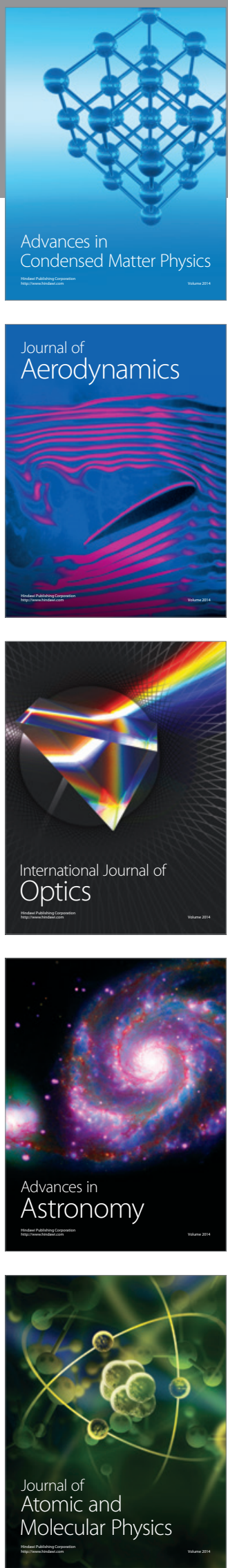